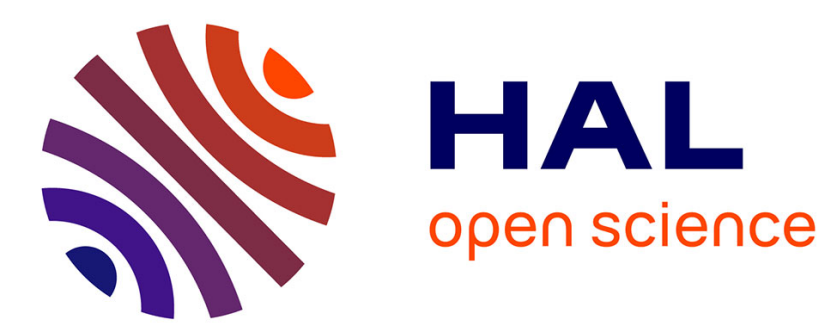

\title{
Parcours moyen des fragments de fission dans quelques métaux avec le mica comme détecteur
}

\author{
J. Mory
}

\section{To cite this version:}

J. Mory. Parcours moyen des fragments de fission dans quelques métaux avec le mica comme détecteur. Revue de Physique Appliquée, 1968, 3 (4), pp.387-395. 10.1051/rphysap:0196800304038700 . jpa00242876

\section{HAL Id: jpa-00242876 https://hal.science/jpa-00242876}

Submitted on 1 Jan 1968

HAL is a multi-disciplinary open access archive for the deposit and dissemination of scientific research documents, whether they are published or not. The documents may come from teaching and research institutions in France or abroad, or from public or private research centers.
L'archive ouverte pluridisciplinaire HAL, est destinée au dépôt et à la diffusion de documents scientifiques de niveau recherche, publiés ou non, émanant des établissements d'enseignement et de recherche français ou étrangers, des laboratoires publics ou privés. 


\title{
PARGOURS MOYEN DES FRAGMENTS DE FISSION DANS QUELQUES MÉTAUX AVEG LE MIGA GOMME DÉTEGTEUR
}

\author{
Par J. MORY, \\ S.M.Pu, Centre d'Études Nucléaires, 92-Fontenay-aux-Roses. \\ (Reçu le 14 juin 1968.)
}

Résumé. - Dans le but d'effectuer le dosage d'éléments fissiles dans les alliages, au moyen des traces de fragments de fission dans le mica, on a mesuré le parcours moyen des fragments de fission dans un certain nombre d'éléments. On trouve, pour l'état écroui, en $\mathrm{mg} . \mathrm{cm}^{-2}$ :

$$
\begin{array}{lll}
\mathrm{Al}: 2,84 ; & \mathrm{Ti}: 3,82 ; & \mathrm{Fe}: 4,22 \\
\mathrm{Ni}: 4,40 ; & \mathrm{Cu}: 5,08 ; & \mathrm{Mo}: 5,18 \\
\mathrm{Ag}: 5,40 ; & \mathrm{Au}: 8,50 . &
\end{array}
$$

On montre qu'il faut tenir compte de l'état cristallin de l'alliage : en général, un recuit augmente les parcours précédents.

Abstract. - In order to evaluate low concentration of fissile elements in alloys by means of fission fragment tracks in mica, the mean range of fission fragments is measured in a number of elements. It is found, for cold worked metals, in $\mathrm{mg} \cdot \mathrm{cm}^{-2}$ :

$$
\begin{array}{lll}
\mathrm{Al}: 2.84 ; & \mathrm{Ti}: 3.82 ; & \mathrm{Fe}: 4.22 \\
\mathrm{Ni}: 4.40 ; & \mathrm{Cu}: 5.08 ; & \mathrm{Mo}: 5.18 \\
\mathrm{Ag}: 5.40 ; & \mathrm{Au}: 8.50 . &
\end{array}
$$

It is shown that one has to take into account the crystalline state of the alloy : generally, annealing increases the above ranges.

I. Introduction. - Une des nombreuses applications de l'enregistrement des particules chargées dans les isolants concerne l'étude de la diffusion à l'état solide de l'uranium ou du plutonium : c'est la méthode fissiographique $[1,2,3]$. Rappelons qu'elle consiste à appliquer l'alliage étudié contre un isolant (mica, plastique, verre), et à irradier l'ensemble dans un flux de neutrons thermiques. L'élément fissile dissous dans l'alliage subit la fission, et chaque fragment de fission atteignant l'isolant y laisse une région endommagée que l'on peut rendre visible au microscope optique au moyen d'une attaque chimique appropriée $[4,5]$. La densité $D$ des traces ainsi formées est liée à la concentration atomique en éléments fissiles par la relation :

$$
D=\frac{1}{2} N_{0} C \sigma_{\mathrm{f}} R_{0} \varphi_{\mathrm{t}}
$$

où $D=$ densité des traces par $\mathrm{cm}^{2}$;

$N_{0}=$ nombre d'atomes de l'alliage par $\mathrm{cm}^{3}$;

$C=$ concentration tomique de l'élément fissile dans l'alliage;

$\sigma_{\mathrm{f}}=$ section efficace microscopique de fission de l'élément fissile; $\varphi_{\mathrm{t}}=$ fluence de neutrons thermiques;

$R_{0}=$ parcours moyen $($ en $\mathrm{cm})$ des fragments de fission dans l'alliage.

Une mesure de $D$ permet donc, en principe, d'accéder facilement à l'inconnue $C$. On voit que, en dehors de $\varphi_{t}$, un paramètre important pour le calcul de $C$ est le parcours $R_{0}$ des fragments de fission dans l'alliage. On peut admettre en toute rigueur que ce parcours (de même que $N_{0}$ ) est le même que dans l'élément de base pur, puisque la méthode est surtout utilisée pour des alliages à très grande dilution en élément fissile $\left(C \leqslant 10^{-6}\right.$ at/at).

Cette méthode a été utilisée pour la première fois lors de la détermination de la concentration en uranium dans le mica [6], puis lors de l'étude de la diffusion du plutonium dans le magnésium [7], de l'uranium dans le titane et le fer [3], et l'aluminium [8,9].

Cependant, le paramètre $R_{0}$ est malheureusement souvent mal connu, ce qui peut entraîner une erreur relativement importante sur $C$. Nous avons donc mesuré $R_{0}$ pour un certain nombre de métaux. De plus, comme $R_{0}$ peut croître notablement s'il y a canalisation, il nous a semblé utile de déterminer $R_{\mathbf{0}}$ 
pour divers états cristallins des différents métaux étudiés, l'obstruction due aux défauts cristallins $[10,11]$ pouvant faire varier $R_{0}$. Notre travail a donc été effectué sur des métaux de textures différentes, la variation des textures ayant été obtenue par des recuits de feuilles minces, à l'origine brutes de laminage, donc très écrouies.

Le seul détecteur utilisé dans ce travail a été le mica muscovite, car il nous semble être le plus intéressant lors de la mesure de très faibles concentrations en éléments fissiles. Dans ce cas, en effet, il est très souvent nécessaire d'utiliser de fortes fluences de neutrons thermiques qui détruisent les plastiques et brunissent les verres, alors que le mica peut supporter facilement des fluences de l'ordre de $10^{19} \mathrm{n} . \mathrm{cm}^{-2}$. Par contre, le mica est un peu moins sensible que les plastıques.

Nous avons ainsi déterminé le parcours moyen des fragments de fission dans les éléments suivants : $\mathrm{Al}, \mathrm{Ti}, \mathrm{Fe}, \mathrm{Ni}, \mathrm{Cu}, \mathrm{Mo}, \mathrm{Ag}, \mathrm{Au}$.

II. Méthode expérimentale. - a) Dispositif ExPÉRIMENTAL. - Nous avons utilisé la méthode classique de l'absorbeur mince [12]. Le dispositif expérimental est représenté sur la figure 1 .

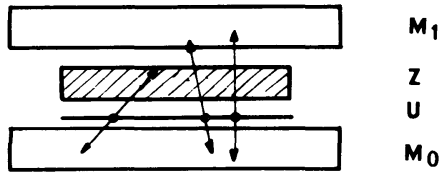

FIG. 1. - Dispositif expérimental :

$\mathrm{M}_{0}$ : mica témoin.

$\mathrm{U}$ : couche mince d'uranium naturel d'épaisseur $t$.

$Z$ : élément absorbeur d'épaisseur $e$.

$M_{1}$ : mica de mesure.

Sur le mica étalon $\mathbf{M}_{0}$ est déposée, par évaporation sous vide, une couche mince (100 à $200 \AA$ ) d'uranium naturel. Une feuille mince de l'absorbeur étudié est interposée entre le mica $M_{0}$ et le mica de mesure $M_{1}$. Le tout, bien serré, est soumis à une irradiation appropriée aux neutrons thermiques, c'est-à-dire telle que la densité des traces sur le mica $M_{0}$ soit maximale, tout en restant facilement dénombrable. Nous avons adopté, pour une densité d'environ $10^{6}$ traces. $\mathrm{cm}^{-2}$, une fluence de $2 \times 10^{12}$ neutrons. $\mathrm{cm}^{-2}$.

b) Préparation des Échantillons. - Mica : il s'agit de mica muscovite. Les échantillons sont des carrés de $2 \times 2 \mathrm{~cm}$, d'épaisseur comprise entre 50 et $100 \mu$. On dépose sur Mo, par évaporation sous vide, une couche mince (100 à $200 \AA)$ d'uranium naturel sur un carré de $1 \mathrm{~cm}^{2}$ au centre de l'échantillon. Les faces en regard des deux micas sont des faces fraîchement clivées.

- Absorbeurs : il s'agit de feuilles minces du commerce, brutes de laminage. Leur épaisseur est telle qu'elles peuvent, en général, servir directement à la mesure. Dans le cas du molybdène et de l'or, nous avons aminci les feuilles en notre possession par polissage électrochimique. Les épaisseurs massiques des feuilles, amincies ou non, ont été déterminées par pesée. Les inhomogénéités d'épaisseur se produisant éventuellement, notamment dans les feuilles amincies, n'ont que peu d'importance; en effet, nous verrons que le coefficient de transmission, point de départ du calcul du parcours moyen, varie linéairement avec l'épaisseur de l'absorbeur. Il suffit donc de connaître avec une bonne précision l'épaisseur moyenne des échantillons.

c) Dénombrement des traces. - Un paramètre important est la dimension des traces sur le mica $\mathbf{M}_{1}$. En effet, les fragments de fission atteignant ce mica ont un parcours résiduel très faible, et laissent des traces qui sont en réalité des points, difficilement différenciables des défauts naturels du mica. Heureusement, on sait que les dimensions transversales des traces de particules chargées varient linéairement avec le temps d'attaque [5]. Pour rendre visibles ces points en tant que traces, nous avons systématiquement attaqué tous nos micas pendant 2 heures dans l'acide fluorhydrique à $40 \%$. Ces points deviennent alors des figures de corrosion en forme de losange ( fig. 2) que l'on ne peut alors plus confondre avec les autres défauts du mica. On vérifie facilement, sur un mica non irradié, qu'une telle attaque ne produit pas de

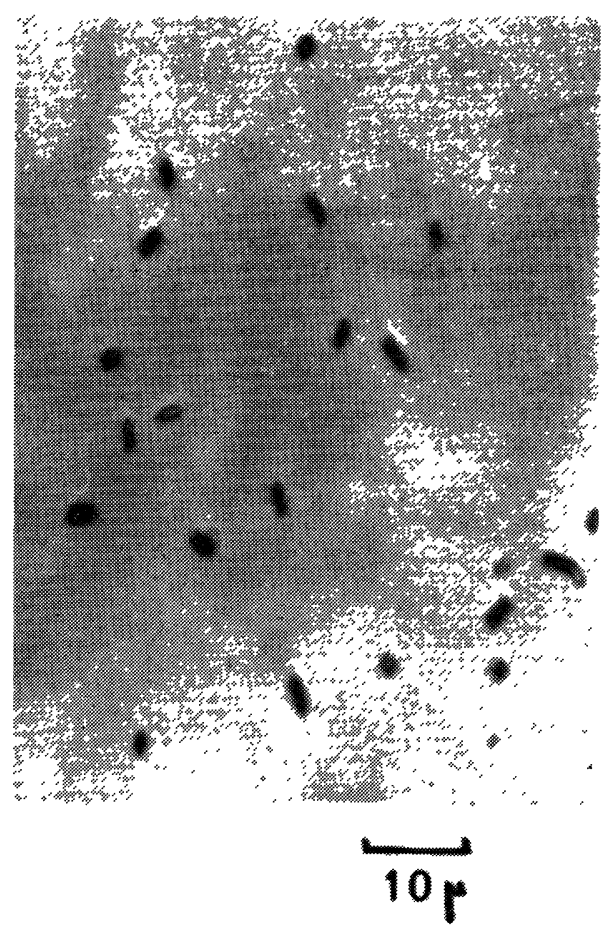

Fig. 2. - Traces de fragments de fission sur un mica $\mathbf{M}_{\mathbf{1}}$. Les fragments de fission ont, ici, été absorbés par 4,46 $\mathrm{mg} \cdot \mathrm{cm}^{-2}$ (ou 2,3 microns) d'or. Attaque : 2 heures dans FH à $40 \%$. 
telles figures, sauf pour les traces fossiles. Ces traces fossiles, de même que les traces de fond provoquées lors de l'irradiation par la fission des noyaux d'uranium en impureté dans le mica, sont par ailleurs peu nombreuses $\left(\simeq 100 \mathrm{t} . \mathrm{cm}^{-2}\right)$ et n'introduisent qu'une erreur négligeable sur nos mesures.

L'épaisseur des sources d'uranium (100 à $200 \AA$ ) et les fluences de neutrons thermiques utilisées ( 2 à 2,5 $\times 10^{12} \mathrm{n} . \mathrm{cm}^{-2}$ ) produisent des densités de traces sur le mica étalon comprises entre $5 \times 10^{5}$ et $10^{6} \mathrm{t} . \mathrm{cm}^{-2}$. Le coefficient de transmission mesuré le plus faible est d'environ 0,1 , ce qui correspond à une densité minimale de traces sur le mica de mesure $\mathbf{M}_{1}$ de $5 \times 10^{4} \mathrm{t} . \mathrm{cm}^{-2}$, densité assez facilement mesurable au microscope optique avec un grandissement de 1000 .

Ces dénombrements s'effectuent sur un verre dépoli du microscope, et, pour éviter toute erreur provenant de grandissements différents, nous avons effectué tous nos comptages avec ce même grandissement de 1000 . Étant donné que les densités sont très différentes d'un mica à l'autre (notamment entre micas $M_{0}$ et $M_{1}$ ), nous avons simplement compté plus de champs de vue pour les micas à faible densité que pour ceux à forte densité. Nos comptages, de plus, ont toujours porté sur plus de 2000 traces pour obtenir une bonne précision statistique. Enfin, ces comptages ont toujours concerné l'ensemble de la surface couverte par l'absorbeur.

III. Définition du parcours moyen des fragments de fission. - Soient $D_{0}$ et $D_{1}$ les densités de traces sur les micas $\mathbf{M}_{\mathbf{0}}$ et $\mathbf{M}_{1}$, l'absorbeur $\mathrm{Z}$ ayant une épaisseur $e$. Le parcours moyen des fragments de fission dans l'élément $\mathrm{Z}$ sera alors donné par la relation [12] :

$$
R=\frac{D_{0}}{D_{0}-D_{1}} e=\frac{e}{1-T}
$$

où $T=D_{1} / D_{0}$ est le coefficient de transmission.

En réalité, la définition du parcours moyen n'est pas la même dans le cas de l'absorbeur mince et dans celui d'une source épaisse (cas des alliages). Ceci est facilement compréhensible, puisque, dans le cas de l'absorbeur mince, la source de fragments de fission est répartie à la surface de cet absorbeur, c'est-à-dire à une distance constante du détecteur. Par contre, dans le cas de la source épaisse, les fragments de fission prennent naissance dans la masse de la source, c'est-àdire à une distance quelconque du détecteur. Ces deux cas sont distingués en annexe et nous nous contenterons de donner ici le résultat : dans le cas de l'absorbeur mince, nous mesurons la moyenne harmonique $\bar{R}_{\mathrm{H}}$ des différents parcours, donnée par :

$$
\frac{2}{\bar{R}_{\mathrm{H}}}=\sum_{i} \frac{\rho_{i}}{R_{i}}
$$

tandis que pour le problème de la source épaisse nous avons besoin de connaître la moyenne arithmétique $\bar{R}_{\mathrm{A}}$ :

$$
\bar{R}_{\mathrm{A}}=\frac{\sum_{i} \rho_{i} R_{i}}{\sum_{i} \rho_{i}}
$$

où $R_{i}$ est le parcours moyen d'un fragment $i$ de rendement de fission $\rho_{i}$.

Mais ces parcours sont peu différents l'un de l'autre. En effet, on peut assimiler l'ensemble des fragments de fission à deux catégories distinctes : les fragments moyens lourds $\left({ }^{140} \mathrm{Ba}\right)$ et les fragments moyens légers ( $\left.{ }^{99} \mathrm{Mo}\right)$ de rendement $\rho_{\mathrm{Ba}}=\rho_{\mathrm{Mo}}=1$. Dans l'aluminium, leurs parcours respectifs ont été mesurés et sont égaux à 3 et $4 \mathrm{mg} \cdot \mathrm{cm}^{-2}$ [13]. On aura donc :

$$
\begin{gathered}
\bar{R}_{\mathrm{A}}=\frac{3+4}{2}=3,5 \mathrm{mg} \cdot \mathrm{cm}^{-2} \\
\frac{2}{\bar{R}_{\mathrm{H}}}=\frac{1}{3}+\frac{1}{4} \quad \text { d'où } \quad \bar{R}_{\mathrm{H}}=3,43 \mathrm{mg} \cdot \mathrm{cm}^{-2}
\end{gathered}
$$

on voit donc que le parcours $\bar{R}_{\mathrm{H}}$ sera inférieur d'environ $2 \%$ au parcours $\bar{R}_{\mathrm{A}}$, et il faudra en tenir compte si l'on veut passer d'un parcours à l'autre en conservant une bonne précision.

\section{Parcours moyen dans les métaux écrouis. -} Nous avons vu que le parcours moyen $\bar{R}_{\mathrm{H}}$, l'épaisseur $e$ d'absorbeur et le coefficient de transmission $T=\frac{D_{1}}{D_{0}}$ sont reliés par la relation $\bar{R}_{\mathrm{H}}=\frac{e}{1-T}$.

Il suffit donc d'une seule détermination de $T$ pour obtenir le parcours moyen. C'est ce que nous avons fait pour la plupart des éléments étudiés, et les résultats figurent dans le tableau I. Sur ce même tableau, nous avons fait figurer les mesures relatives à la référence [14].

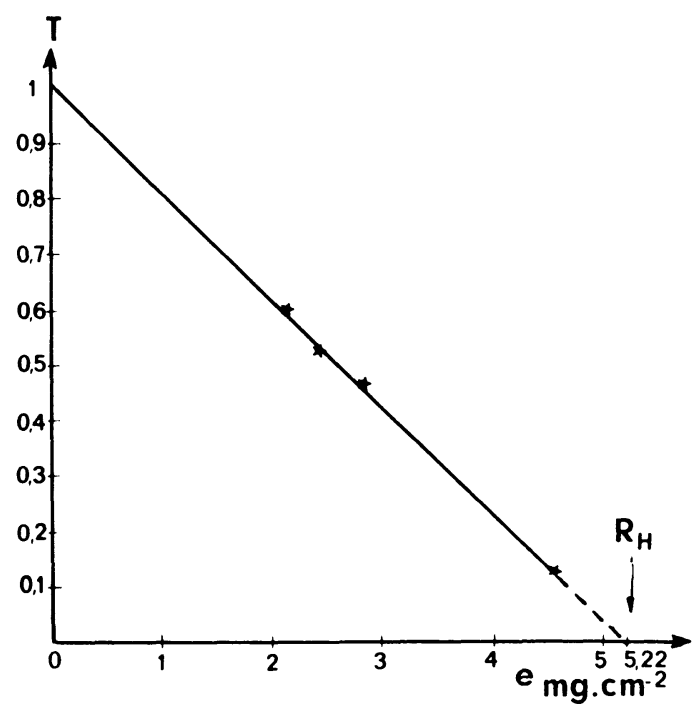

Fig. 3. - Variation du coefficient de transmission en fonction de l'épaisseur de molybdène. 
TABLEAU I

Pargours des fragments de fission DANS DIFFÉRENTS MÉTAUX BRUTS D'ÉGROUISSAGE

\begin{tabular}{|c|c|c|c|c|c|}
\hline ÉLÉMENTS & $\begin{array}{l}\text { ÉPAISSEUR } \\
\left(\mathrm{mg} \cdot \mathrm{cm}^{-2}\right)\end{array}$ & $T$ & $\begin{array}{l}\text { PARGOURS } \\
\left(\mathrm{mg} \cdot \mathrm{cm}^{-2}\right)\end{array}$ & $\begin{array}{l}\text { PARGOURS } \\
\text { (microns) }\end{array}$ & $\begin{array}{c}\text { PARGOURS } \\
\text { réf. [14] } \\
\left(\mathrm{mg} . \mathrm{cm}^{-2}\right.\end{array}$ \\
\hline - & - & - & - & - & - \\
\hline $\mathrm{Al}$ & 1,20 & 0,575 & 2,85 & 10,54 & 3,0 \\
\hline $\mathrm{Al}$ & 1,345 & 0,520 & 2,83 & 10,47 & 3,0 \\
\hline $\mathrm{Ti}$ & 2,25 & 0,410 & 3,82 & 8,50 & - \\
\hline $\mathrm{Fe}$ & 2,42 & 0,422 & 4,22 & 5,36 & - \\
\hline $\mathrm{Ni}$ & 3,31 & 0,246 & 4,40 & 5,07 & 4,0 \\
\hline $\mathrm{Cu}$ & 2,44 & 0,515 & 5,08 & 5,71 & - \\
\hline Mo & (1) & (1) & 5,18 & 5,08 & - \\
\hline $\mathrm{Ag}$ & 3,71 & 0,313 & 5,40 & 5,14 & 5,4 \\
\hline $\mathrm{Au}$ & $(2)$ & (2) & 8,50 & 4,41 & 9,2 \\
\hline
\end{tabular}

(1) Voir figure 3 .

(2) Voir figure 4 .

Les valeurs de la dernière colonne évaluées à partir d'une courbe $R=f(Z)$ fournie par les auteurs [14]. $Z=$ numéro atomique.

Pour vérifier la validité de la méthode, nous avons mesuré le parcours pour 4 épaisseurs différentes de molybdène et d'or. En portant, sur un graphique, $T$ en fonction de $e$, on doit obtenir une droite passant par $T=1$ pour $e=0$, et par $T=0$ pour $e=\bar{R}_{\mathrm{H}}$. Ce dernier point sera évidemment obtenu par extrapolation de la droite expérimentale. On voit sur les figures 2 et 3 que la dispersion des points est très faible, d'environ $1 \%$ par rapport à la droite moyenne.

Nous avons également tenu compte du fait que la source de fragments de fission n'est pas infiniment mince. Si $t$ est l'épaisseur de la couche d'uranium, la relation complète devient :

$$
\bar{R}_{\mathrm{H}}=\frac{1}{1-T}\left(e+\frac{1}{2} c t\right)
$$

où $c$ est théoriquement le pouvoir d'arrêt relatif de l'uranium par rapport à l'élément étudié [15]. En l'absence de certitude sur ce point, et $c$ étant toujours voisin de l'unité, nous avons pris arbitrairement $c=1$. Ce choix n'influe que peu sur le résultat, étant donné la grande différence entre $e$ et $t$. Enfin, $t$ nous est donné directement par la densité $D_{0}$ des traces sur le mica $\mathrm{M}_{0}$.

Discussion. - Nous avons porté sur la figure 5 la variation du parcours moyen $\bar{R}_{\mathrm{H}}$ des fragments de fission en fonction du numéro atomique des différents absorbeurs. On voit qu'un certain nombre de parcours (dans $\mathrm{Al}, \mathrm{Ti}, \mathrm{Fe}, \mathrm{Ni}, \mathrm{Au}$ ) s'alignent correctement sur une courbe continue monotone, ce à quoi l'on pouvait s'attendre. Par contre, on constate que certains points s'en écartent. Ainsi le parcours dans le

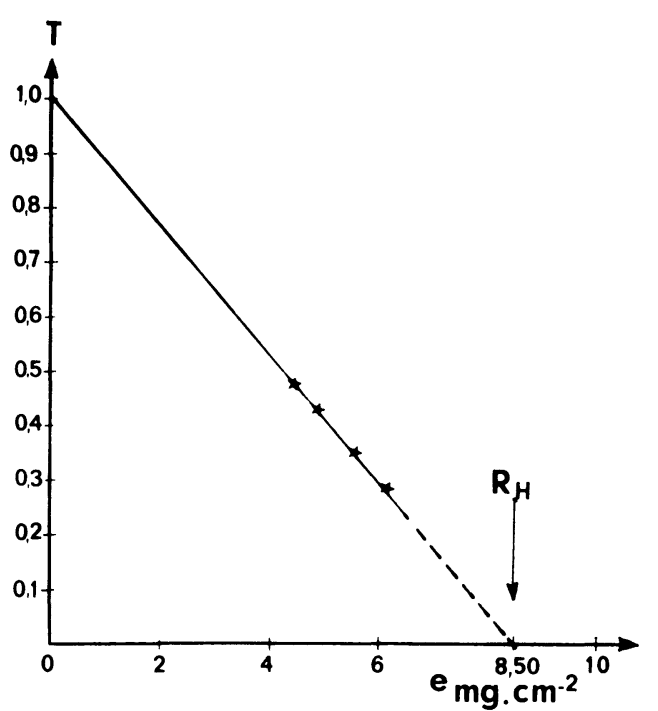

FIG. 4. - Variation du coefficient de transmission en fonction de l'épaisseur d'or.

cuivre semble « anormalement " grand, tandis que ceux dans le molybdène et l'argent sont faibles.

De récentes mesures de certains de ces parcours ont été effectuées par Abdullaev et al. [14] au moyen d'une source de curium. Les résultats obtenus avec le mica ne semblent pas mettre en évidence un parcours particulier pour l'argent. Quant au cuivre et au molybdène, ils n'ont pas été étudiés dans ce travail. Par contre, les auteurs de la référence [12] ne semblent pas avoir relevé d'anomalie pour le cuivre. 
V. Parcours moyen dans les métaux recristallisés. - Dans la plupart des déterminations de parcours de particules chargées, on considérait jusqu'à présent l'élément absorbeur comme un corps amorphe. Or, on sait maintenant que les métaux, et les corps cristallins en général, présentent des directions privilégiées, parallèles aux axes denses, et suivant lesquelles une particule chargée subit un ralentissement moindre et voit ainsi son parcours allongé. C'est le phénomène de « canalisation », étudié théoriquement [16] et expérimentalement [17]. La canalisation a déjà été utilisée avec succès lors de l'étude de la transmission des particules $\alpha$ à travers des absorbeurs minces [1, 10,11], et a permis de mettre en évidence de fortes anisotropies de transmission selon la texture, la taille des grains, la présence de dislocations ou d'impuretés, etc. Il nous a semblé intéressant d'étudier également l'influence

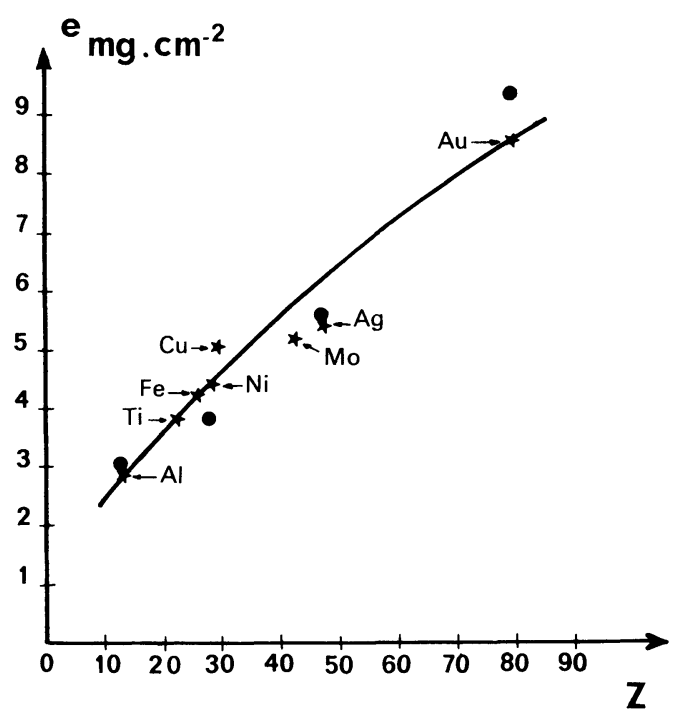

FrG. 5. - Variation du parcours des fragments de fission en fonction du numéro atomique $Z$ de l'absorbeur :

$\star$ : nos résultats,

- : résultats de la référence [14]. de l'état cristallin des absorbeurs minces sur le parcours des fragments de fission. Pour cela, nous avons fait subir aux métaux déjà étudiés des recuits plus ou moins intenses. Il ne faut pas nous attendre, en général, à des variations considérables du parcours, puisque ce phénomène de canalisation n'affecte qu'un nombre très restreint de particules, c'est-à-dire celles qui abordent le cristal dans des directions très proches de celles de canalisation. Néanmoins, nous verrons que cet effet est visible.

a) Aluminium. - Les feuilles minces utilisées sont les mêmes que celles étudiées précédemment, mais recuites 15 heures à $600^{\circ} \mathrm{C}$, et sont ainsi complètement recristallisées. On voit sur le tableau II que le parcours est ici $\bar{R}_{\mathrm{H}}=3,26 \mathrm{mg} \cdot \mathrm{cm}^{-2}$, contre $\bar{R}_{\mathrm{H}}=2,84 \mathrm{mg} \cdot \mathrm{cm}^{-2}$ pour l'aluminium écroui, soit une augmentation de $11 \%$.

b) Molybdìne. - Une feuille d'épaisseur $4,51 \mathrm{mg} \cdot \mathrm{cm}^{-2}$ a été recuite sous vide 1 heure à

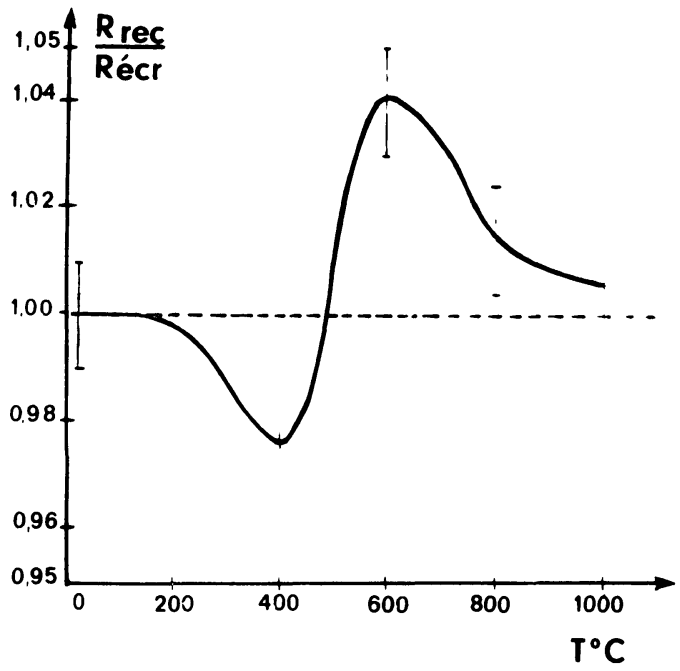

FIG. 6. - Variation du parcours relatif des fragments de fission dans l'or en fonction de la température de recuit (durée de chaque recuit : $1 \mathrm{~h}$ ).

TABLEAU II

PARgours DES FRAGMents DE FISSION DANS DIFFÉRENTS ÉLÉMENTS AYANT SUBI UN REGUIT

\begin{tabular}{cccccc} 
ÉLÉments & $e\left(\mathrm{mg} \cdot \mathrm{cm}^{-2}\right)$ & \multicolumn{2}{c}{ ReGuit } & $\bar{R}_{\mathrm{H}}\left(\mathrm{mg} \cdot \mathrm{cm}^{-2}\right)$ & $\bar{R}_{\mathrm{H}}$ (microns) \\
- & - & \multicolumn{2}{c}{-} & - & - \\
$\mathrm{Al}$ & 1,2 & $15 \mathrm{~h}$ à & $600^{\circ} \mathrm{C}$ & 3,26 & 12,07 \\
& 1,345 & $15 \mathrm{~h}$ à & $600^{\circ} \mathrm{C}$ & 3,27 & 12,11 \\
$\mathrm{Mo}$ & 4,51 & $1 \mathrm{~h}$ à $1600^{\circ} \mathrm{C}$ & 5,49 & 5,38 \\
$\mathrm{Au}$ & 4,875 & $1 \mathrm{~h}$ à & $400^{\circ} \mathrm{C}$ & 8,28 & 4,29 \\
& 4,46 & $1 \mathrm{~h}$ à $600^{\circ} \mathrm{C}$ & 8,84 & 4,58 \\
& 5,56 & $1 \mathrm{~h}$ à $800^{\circ} \mathrm{C}$ & 8,58 & 4,45 \\
& 6,13 & $1 \mathrm{~h}$ à $1000^{\circ} \mathrm{C}$ & 8,63 & 4,47
\end{tabular}


TABLEAU III

Abondances Relatives des différentes teXtures De L'OR SELON LE ReGUit SUbi $\langle 110\rangle$ DÉSIGNE LES DIREGTIONS $\langle 110\rangle$ PERPENDIGULAIRES A LA LAME MÉTALLIQUE

$\begin{array}{lccccc} & \text { Écrour } & 400^{\circ} \mathrm{C} & 600{ }^{\circ} \mathrm{C} & 800^{\circ} \mathrm{C} & 1000^{\circ} \mathrm{C} \\ \langle 110\rangle & - & - & - & - & - \\ \langle 100\rangle & 7,5 & 0 & 25 & 14 & 0 \\ \langle 111\rangle & 185 & 58 & 30 & 36 & 0 \\ & 0 & 17 & 40 & 28 & 45\end{array}$

environ $1600^{\circ} \mathrm{G}$, par effet Joule. Le parcours $\left(5,49 \mathrm{mg} . \mathrm{cm}^{-2}\right.$ contre $5,18 \mathrm{mg} \cdot \mathrm{cm}^{-2}$ pour le métal écroui) subit une augmentation de 5,5\%.

c) OR. - Nous avons repris les mêmes échantillons amincis qui nous avaient servi à déterminer le parcours dans l'or écroui. Nous leur avons fait subir des recuits de 1 heure à différentes températures; les résultats obtenus sont portés sur le tableau II. Nous avons d'autre part tracé sur la figure 6 la variation $\mathrm{du}$ parcours relatif (quotient du parcours dans l'or recuit et du parcours dans l'or écroui).

d) Discussion. - Les expériences de transmission des particules $\alpha[18,19]$ ont montré qu'un métal recristallisé était plus transparent que le même métal brut de laminage. Cela provient en particulier de ce que, dans un métal recuit, la plupart des défauts (défauts ponctuels, dislocations, etc.) sont éliminés et, surtout, que, les grains étant plus gros, l'importance des joints de grains, opaques aux particules chargées, diminue.

Ceci explique l'augmentation sensible du parcours moyen mesuré dans l'aluminium et le molybdène.

Le cas de l'or est plus complexe, et, ici, intervient sans doute l'effet de la texture cristalline. Une étude sommaire par rayons $\mathrm{X}$ nous a permis de connaître, approximativement, le nombre de cristaux ayant un plan cristallographique donné parallèle à la surface de l'échantillon dans des lames d'or de 5 microns ayant subi le même recuit que celles utilisées. Le tableau III donne, en unités arbitraires, ce nombre pour les principales directions.

Si l'on se rappelle que la direction la plus favorable, dans un métal cubique faces centrées, est $\langle 110\rangle$ suivie de $\langle 100\rangle$, puis de $\langle 111\rangle$, on voit que la variation de parcours des fragments de fission semble liée à l'évolution de la texture de l'or : maximale en même temps que la texture $\langle 110\rangle$, minimale quand les textures $\langle 100\rangle$ ou $\langle 111\rangle$ dominent. Il faut évidemment ajouter à ces variations de texture le grossissement des grains qui, lui, tendra toujours à augmenter le parcours. Il ne s'agit, pour le moment, que d'une explication sommaire. En particulier, le fait que les parcours moyens dans l'or écroui et l'or recuit à $1000^{\circ} \mathrm{C}$ soient sensiblement égaux est probablement fortuit. e) Ganaligraphie Par fragments de fission. Nous avons conservé le montage de la figure 1, en remplaçant l'uranium naturel par de l'uranium enrichi, et l'absorbeur Z par un absorbeur d'épaisseur correspondant approximativement au parcours maximal des fragments de fission dans l'élément étudié. Une irradiation aux neutrons thermiques de $5 \times 10^{16} \mathrm{n} . \mathrm{cm}^{-2}$, soit une irradiation aux fragments de fission d'environ $5 \times 10^{11}{\mathrm{f} . \mathrm{cm}^{-2}}$, fait apparaître sur le mica $M_{1}$ une image représentée figure 7. L'absorbeur est ici

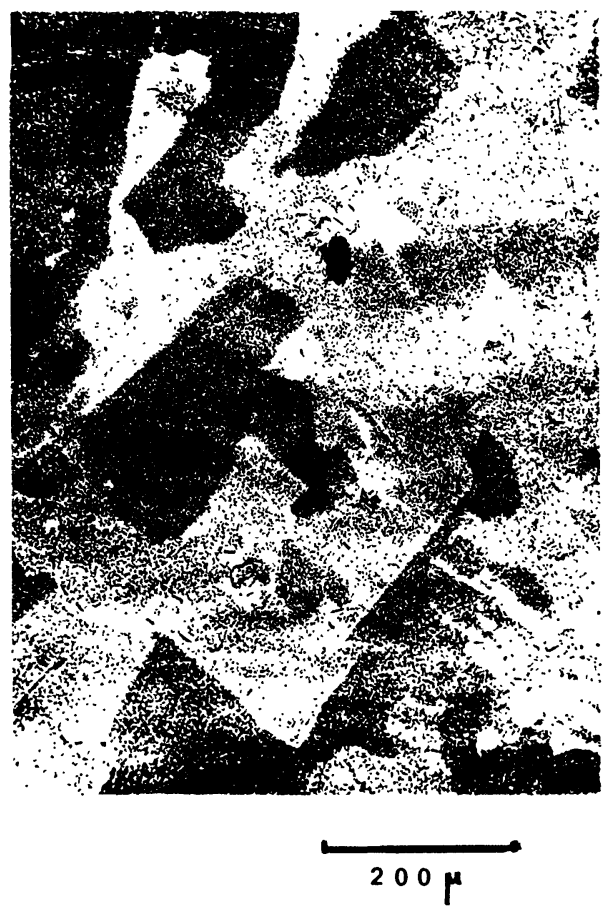

FIG. 7. - Canaligraphie par fragments de fission d'une lame de platine de $7 \mu$, recuite $15 \mathrm{~h}$ à $950^{\circ} \mathrm{C}$. On voit nettement apparaître différentes zones dont le noircissement dépend de l'orientation de chaque grain.

une lame de platine de $7 \mu$ (soit environ $14 \mathrm{mg} \cdot \mathrm{cm}^{-2}$ ) recuite 15 heures à $950 \circ \mathrm{C}$. A la suite de Quéré [11], nous appellerons cette image formée de particules canalisées "Canaligraphie par fragments de fission ". 
Une comparaison entre canaligraphie et micrographie montre que chaque tache noire, grise ou blanche de la canaligraphie correspond à un grain bien déterminé de la micrographie. On voit ainsi que tous les grains n'ont pas le même coefficient de transmission, et donc que le parcours des fragments de fission varie le long de l'échantillon. On peut supposer que, comme dans le cas des particules $\alpha$ [17], le coefficient de transmission moyen, donc le parcours sont fonction du recuit, ce qui explique les variations du parcours dans l'or constatées dans nos expériences. Une description plus détaillée de ces images dans le cas du platine et du tungstène est donnée ailleurs [20].

VI. Validité des résultats pour les mesures de concentrations. - Le but de nos mesures était de fournir des parcours moyens directement applicables dans la formule (1). Les valeurs que nous avons trouvées constituent une bonne première approximation. Un certain nombre de remarques vont nous montrer les limites de cette approximation :

a) Les parcours dans les sources épaisses sont d'environ $2 \%$ plus grands que ceux dans les absorbeurs minces, par suite de leur définition mathématique différente.

b) Les alliages que nous aurons à étudier sont fortement recuits, ne serait-ce que pour en permettre l'homogénéisation ou la diffusion. Dans ce cas, la texture et la taille des grains peuvent être différentes de celles des feuilles minces utilisées ici; nos résultats montrent que les valeurs de $R$ dépendent de la texture de manière non négligeable.

Deux phénomènes, au moins, sont ici en cause :

- La canalisation : les grains dans un échantillon massif étant en général plus gros que dans une feuille mince, l'effet d'obstruction de la canalisation par les joints de grains sera beaucoup plus faible dans le cas de l'alliage épais que dans celui de l'absorbeur mince;

- Le blocage : dans un alliage dilué, les atomes du soluté, s'ils sont substitutionnels, occupent des sites normaux du réseau. Si un tel atome subit la fission, les fragments produits ont donc peu de chance d'être canalisés. Ils risquent, au contraire, d'être « bloqués » dans la rangée où ils ont pris naissance [21]. Leur parcours sera ainsi réduit, et cela avec une probabilité d'autant plus grande que leur direction de propagation initiale semblait plus favorable à leur canalisation.

Ces deux phénomènes : canalisation et blocage, agissent donc en sens inverse, sans que l'on puisse même dire a priori lequel sera prépondérant.

c) Il serait hasardeux de lire sur la courbe 5 une valeur de $R$ pour un élément non étudié en se servant des valeurs expérimentales d'éléments voisins dans la classification périodique. On trouve un exemple frappant de cette limitation dans le cas du cuivre, dans lequel le parcours est nettement supérieur à celui du nickel, bien que les numéros atomiques des deux absorbeurs ne diffèrent que d'une unité.

VII. Conclusion. - Nous venons de voir (chap. V) que les parcours déterminés par la méthode de l'absorbeur mince peuvent être utilisés, mais avec précaution, lorsque l'on passe aux sources épaisses, cas des alliages. Il est certain qu'il aurait été plus valable d'étudier directement les parcours dans des alliages métaluranium. Mais cette méthode est plus lourde, car elle nous oblige à des mesures absolues de la concentration en uranium (difficile à déterminer avec précision, par les méthodes classiques, dans le cas des alliages très dilués), et de la fluence de neutrons thermiques (rarement connue à mieux que $5 \%$ près). Néanmoins, nous comptons élaborer un tel alliage, et y étudier la variation du parcours moyen des fragments de fission en fonction de la texture cristalline.

D'autre part, ce travail nous a permis de mettre en évidence les possibilités de canaligraphie de feuilles minces par fragments de fission. Il semble que cette méthode soit plus fine que la canaligraphie par particules $\alpha$, bien que sa mise en application soit plus difficile.

Ces deux points : variation du parcours des fragments de fission en fonction de la texture dans un métal massif et canaligraphie par fragments de fission, sont en cours d'étude et feront l'objet de communications ultérieures.

\section{ANNEXE}

Parcours moyen des fragments de fission. - Soit, dans un absorbeur $\mathrm{Z}$, un fragment de fission $\mathrm{F}_{i}$, de rendement de fission $\rho_{i}$ et de parcours moyen $R_{i}$.

a) GAS DE L'ABSorbeur mince ( fig. 8 a). - Le fragment $\mathrm{F}_{i}$, émis en $\mathrm{A}$, émergera de l'absorbeur $\mathrm{Z}$

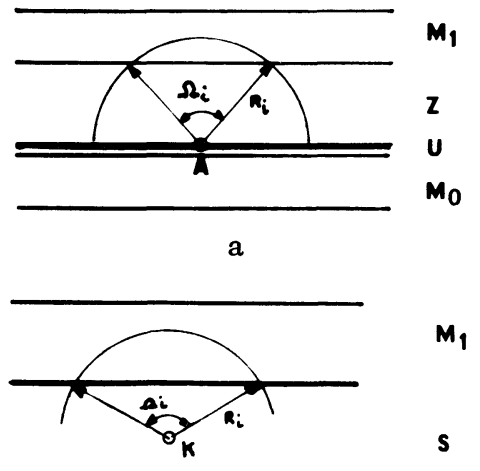

$\mathrm{b}$

FIG. 8. - Mesure du parcours moyen des fragments de fission :

8 a) Dans le cas de l'absorbeur mince.

8 b) Dans le cas de la source épaisse. 
s'il est émis dans l'angle solide $\Omega_{i}$, c'est-à-dire avec une probabilité :

$$
P_{\mathrm{T}}=\frac{\Omega_{i}}{4 \pi}=\frac{1}{2} \cdot \frac{R_{i}-e}{R_{i}} .
$$

Pour l'ensemble $N_{0}$ des fissions, le nombre de fragments émergeant de l'absorbeur sera :

$$
\begin{aligned}
N_{1} & =N_{0} \sum_{i} \frac{1}{2} \rho_{i} \frac{R_{i}-e}{R_{i}} \\
& =\frac{1}{2} N_{0} \sum_{i} \rho_{i}-\frac{1}{2} N_{0} e \sum_{i} \frac{\rho_{i}}{R_{i}}
\end{aligned}
$$

soit, en tenant compte de ce que : $\sum_{i} \rho_{i}=2$ :

$$
N_{1}=N_{0}-\frac{1}{2} N_{0} e \sum_{i} \frac{\rho_{i}}{R_{i}}
$$

Le coefficient d'absorption est alors :

$$
\frac{N_{0}-N_{1}}{N_{0}}=\frac{e}{2} \sum_{i} \frac{\rho_{i}}{R_{i}}
$$

Soit $\vec{R}_{\mathrm{H}}$ la moyenne harmonique de tous les parcours moyens $R_{i}$, définie par :

$$
\frac{2}{\bar{R}_{\mathrm{H}}}=\sum_{i} \frac{\rho_{i}}{R_{i}}
$$

on obtient :

$$
\frac{N_{0}-N_{1}}{N_{0}}=\frac{e}{\bar{R}_{\mathrm{H}}}
$$

ou :

$$
\bar{R}_{\mathrm{H}}=\frac{N_{0}}{N_{0}-N_{1}} e
$$

soit, en passant aux densités de traces sur $M_{0}$ et $M_{1}$ :

$$
\bar{R}_{\mathrm{H}}=\frac{D_{0}}{D_{0}-D_{1}} e \text {. }
$$

b) GAS de la source épaisse (fig. 8 b). - De même que le cas précédent, le fragment $\mathrm{F}_{i}$, émis en $\mathrm{K}$, émergera de la source $\mathrm{S}$ avec une probabilité :

$$
P_{\mathrm{T}}=\frac{1}{2} \cdot \frac{R_{i}-e}{R_{i}} .
$$

Soit $N_{0}$ le nombre total de fissions par $\mathrm{cm}^{3}$ de la source. Le nombre de fissions se produisant dans une couche d'épaisseur de à la distance $e$ du mica $\mathbf{M}_{1}$ sera :

$$
\mathrm{d} N_{0}=N_{0} \mathrm{~d} e .
$$

Le nombre de fragments $\mathrm{F}_{i}$ émis dans la couche de et atteignant le mica sera :

$$
\mathrm{d} N_{i}=\frac{1}{2} N_{0} \rho_{i} \frac{R_{i}-e}{R_{i}} \mathrm{~d} e .
$$

Le nombre total de fragments $F_{i}$ atteignant le mica $\mathbf{M}_{1}$ sera :

$$
N_{i}=\int_{0}^{R_{i}} \mathrm{~d} N_{i}=\frac{1}{4} N_{0} \rho_{i} R_{i} .
$$

Le nombre total de fragments de fission atteignant le mica $\mathbf{M}_{1}$ sera :

$$
N_{1}=\sum_{i} N_{i}=\frac{N_{0}}{4} \sum_{i} \rho_{i} R_{i} .
$$

Soit $\bar{R}_{\mathrm{A}}$ la moyenne arithmétique de tous les parcours moyens $R_{i}$, définie par :

$$
\bar{R}_{\mathrm{A}}=\frac{\sum_{i} \rho_{i} R_{i}}{\sum_{i} \rho_{i}}=\frac{1}{2} \sum_{i} \rho_{i} R_{i} .
$$

Le nombre total de fragments de fission atteignant le mica $\mathrm{M}_{1}$ deviendra :

$$
N_{1}=\frac{1}{2} N_{0} \bar{R}_{\mathrm{A}}
$$

En considérant la densité $D_{1}$ des traces par $\mathrm{cm}^{2}$ sur le mica et le nombre $N_{5}$ d'atomes d'uranium 235 par $\mathrm{cm}^{3}$ d'alliage, la relation définitive devient :

$$
\bar{R}_{\mathrm{A}}=\frac{2 D_{1}}{N_{5} \sigma_{5} \varphi_{\mathrm{t}}}
$$

où $\sigma_{5}=$ section efficace de fission de ${ }^{235} \mathrm{U}$,

$\varphi_{t}=$ fluence de neutrons thermiques.

On voit donc que l'on est conduit à prendre des définitions mathématiques différentes pour le parcours moyen, selon le cas étudié.

\section{BIBI,IOGRAPHIE}

[1] Mory (J.), DE Keroulas (F.), Resneau (J. C.) et QUÉ́RÉ (Y.), VIth Int. Conf. on Corpuscular Photography, Florence, 1966.

[2] DE Keroulas (F.), MORY (J.) et QuÉRÉ (Y.), J. Nucl. Mat., 1967, 22, 276.

[3] DE KERoulas (F.), Rapport C.E.A., R. 3253, 1967.

[4] Silk (E. C. H.) et Barnes (R. S.), Phil. Mag., 1959, 4, 970.
[5] Price (P. B.) et Walker (R. M.), J. Appl. Phys., 1962, 33, 3407.

[6] Price (P. B.) et Walker (R. M.), Appl. Phys. Letters, 1963, 2, 23.

[7] MoRy (J.), Rapport C.E.A., R. 2846, 1965.

[8] Blecheit (J. J.), VAn Craeynest (A.) et CaI,ais (D.), J. Nucl. Mat., 1968 (à paraître).

[9] BLECHE'T (J. J.), Rapport C.E.A., 1968 (à paraître). 
[10] Quéré (Y.), Resneau (J. C.) et MORY (J.), C. R. Acad. Sci. Paris, 1966, 262, 1528.

[11] QUÉRḱ (Y.), J. Physique, 1968, 29, 215.

[12] Segré (E.) et Wiegand (C.), Phys. Rev., 1946, 70, 808.

[13] Aras (N. K.), Menon (M. P.) et Gordon (G. E.), Nucl. Phys., 1965, 69, 337.

[14] Abdullaev (Kh.), Perelygin (V. P.), TretiaKova (S. P.) et Shadieva (N. Kh.), Rapport P 13-3585, J.I.N.R., Dubna, 1967.

[15] Alexander (J. M.) et Gazdik (M. F.), Phys. Rev., $1960,120,874$.
[16] Holmes (D. K.), The interaction of radiations with solids, North-Holland, 1964, p. 33.

[17] Nelson (R. S.) et Thompson (M.), Phil. Mag., 1963, 8, 1677.

[18] Quéré (Y.) et Couve (H.), J. Appl. Phys., 1968, 39, 4012.

[19] Couve (H.), Rapport C.E.A., 1968 (à paraître).

[20] MORy (J.) et DelsarTe (G.), Radiation effects (à paraître).

[21] DomeiJ (B.) et BJöRkøvist (K.), Phys. Letters, 1965, 14, 127. 